\title{
Análise multinivel aplicada aos dados do NELS:88
}

JACOB ARIE LAROS

$\mathrm{UnB}$

JOÁO LUIZ PEREIRA MARCIANO

UnB

\section{RESUMO}

$\mathrm{Na}$ Psicologia eáreas afins, está aumentando a consciência de que o pressuposto da independência das observaçôes da regressão tradicional nem sempre é refletido pela realidade. O objetivo deste artigo é ilustrar a análise multinível, uma alternativa à regressão tradicional, usando um banco de dados de acesso público: o NELS:88 (National Educational Longitudinal Study). NELS:88 é uma pesquisa conduzida pelo Ministério da Educaçáo dos Estados Unidos com o propósito de identificar o efeito de variáveis socioeconômicas e escolares sobre o desempenho educacional. O banco contém dados de 21.580 alunos da oitava série do ensino fundamental, provenientes de 1.003 escolas. Nessa pesquisa, foi aplicado um teste de Matemática, acompanhado por um questionário sobre características socioeconômicas e culturais dos alunos. Pode-se concluir que o banco de dados NELS:88 é muito adequado para ilustrar, de forma prática, como funciona uma análise multinível.

Palavras-chave: análise multinível, modelo hierárquico linear, correlação intraclasse, NELS:88.

\section{RESUMEN}

En Psicología y en áreas afines está aumentando la conciencia de que la realidad no siempre refleja el presupuesto de la independencia de las observaciones de la regresión tradicional.El objetivo de este artículo es ilustrar el análisis multinivel, una alternativa a la regresión tradicional, usando un banco de datos de acceso público, NELS:88 (National Educational Longitudinal Study). El NELS:88 es una investigación conducida por el Ministerio de Educación de los Estados Unidos con el propósito de identificar el efecto de variables socioeconómicas y escolares sobre el desempeńo educativo. El banco contiene datos de 21.580 alumnos de la octava serie de 
la educación fundamental, provenientes de 1.003 escuelas. En la investigación se aplicó un test de Matemática, acompańado de un cuestionario sobre características socioeconómicas y culturales de los alumnos. Se puede concluir que el banco de datos NELS:88 es muy adecuado para ilustrar, de forma práctica, como funciona el análisis multinivel.

Palabras clave: análisis multinivel, modelo jerárquico lineal, correlación intraclase, NELS:88.

\section{ABSTRACT}

In Psychology and related fields there is growing awareness that the independent observations derived traditional regression analysis are not always reflected in reality. The objective of this article is to illustrate multilevel analysis, an alternative to traditional regression analysis, using a dataset of public access, NELS:88. (National Educational Longitudinal Study). NELS:88 is conducted by the Ministry of Education of the United States of America and was designed to identify the effect of socio-economic and school variables on educational performance. The dataset contains data of 21,580 eighth grade students of elementary school distributed amongst 1,003 schools. In the NELS:88 study a mathematics test was administered together with a questionnaire about socio-economic and cultural characteristics of the students. It can be concluded that the NELS: 88 dataset serves very well to illustrate in a practical way how a multilevel analysis is carried out.

Keywords: multilevel analysis, hierarchical linear model, intraclass correlation, NELS:88. 


\section{INTRODUÇÃO}

A regressão múltipla é uma das técnicas de análise de dados mais utilizadas nas áreas de ciências sociais e humanas. O problema maior em usar a regressão múltipla nessas áreas é o fato de que, em muitas ocasióes, um dos pressupostos centrais, a independência das observaçôes, é violado. Nas ciências sociais e humanas, os dados coletados são freqüentemente de pessoas agrupadas em clusters. Uma conseqüência da dependência entre as observações é a subestimação dos erros padrão dos coeficientes da regressão. Uma alternativa para a técnica de regressão múltipla que leva em consideração a estrutura hierárquica dos dados é a análise multinível. Essa técnica é um tipo de análise de regressão que, simultaneamente, leva em consideração múltiplos níveis de agregação, tornando corretos erros padrão, intervalos de confiança e testes de hipóteses. Quando existe uma estrutura hierárquica na população de interesse, a análise multinível é a opção metodologicamente correta para estabelecer as relações entre as variáveis.

A análise multinível também é conhecida como: Modelo Hierárquico Linear, Modelo de Efeitos Mistos, Modelo de Efeitos Aleatórios e Regressão Hierárquica. Ela é uma extensão do modelo de regressão tradicional quando variáveis são analisadas dispostas em vários níveis de agregação. Isso ocorre com freqüência no contexto educacional quando, por exemplo, se deseja estudar o desempenho do aluno com base nas variáveis associadas ao próprio aluno (nível 1), variáveis associadas ao professor ou à turma (nível 2) e variáveis associadas ao diretor ou à escola (nível 3). Ao lidar com variáveis em diferentes níveis, o modelo de regressão tradicional pode não ser o mais adequado, pois não leva em consideraçáo a correlaçáo entre indivíduos associados a um mesmo nível de agregação. É o caso da correlação entre alunos de uma mesma turma ou escola. Quanto maior for a correlação entre indivíduos maior a inadequação do modelo de regressão tradicional.

Análise multinível aplica-se a uma população com estrutura hierárquica. A obtenção de uma amostra de tal população se dá pela escolha aleatória dentre unidades do nível macro (por exemplo, hospitais). Uma vez selecionadas essas unidades, o segundo passo seria escolher, também de modo aleatório, as unidades do nível micro (por exemplo, pacientes dentro dos hospitais). Este procedimento descreve sumariamente a técnica adequada para se conseguir uma amostra na qual o pressuposto de independência entre os sujeitos não é violado; porém, por motivos práticos, de natureza financeira ou logística, o que é feito freqüentemente é proceder a uma amostragem de todos os indivíduos disponíveis, depois de escolhidas as unidades do nível macro. É bom enfatizar que, algumas vezes, nem mesmo a escolha das unida- 
des do nível macro é feita de maneira aleatória, mas de acordo com a conveniência ou disponibilidade de recursos. Obviamente, nesses casos, ocorre a violação do pressuposto de independência das observaçóes, o que pode levar a resultados espúrios.

Os testes estatísticos tradicionais confiam pesadamente no pressuposto da independência das observaçóes. Portanto, o modelo de regressão tradicional pode não ser o mais adequado, pois não leva em consideração a dependência das observaçóes entre os membros de um mesmo grupo. Quanto maior essa dependência, mais a análise multinível se torna necessária. A dependência entre as observaçooes é indicada pela chamada correlaçáo intraclasse, a qual representa a homogeneidade em um mesmo grupo, e, ao mesmo tempo, a heterogeneidade entre grupos distintos.

A fim de ilustrar a dependência entre observaçóes, pode-se citar o exemplo de pesquisas educacionais nas quais alunos são aninhados ou agrupados em escolas. Os alunos de uma mesma escola tendem a ser similares, em razão do processo de seleção por ela empregado (por exemplo, algumas escolas podem atrair alunos de níveis socioeconômicos mais elevados, enquanto outras atraem alunos de níveis socioeconômicos mais baixos), e do ambiente e da história comuns que os alunos compartilham por freqüentar a mesma escola. Estruturas hierárquicas também podem ser encontradas nos estudos transnacionais, nos quais os indivíduos são aninhados em suas unidades nacionais; na pesquisa organizacional, em que os indivíduos são agrupados em departamentos, e estes, por sua vez, em organizaçóes; na pesquisa familiar, com os membros agrupados em famílias; e na pesquisa metodológica, quanto aos efeitos do entrevistador, com os respondentes agrupados por entrevistadores. Exemplos de dados hierárquicos menos óbvios são pesquisas longitudinais e pesquisas de curvas de crescimento, nas quais séries de observaçóes distintas são vistas como aninhadas por indivíduos; e metanálise, em que os objetos de análise são agrupados em diferentes estudos (Hox, 2002).

Nos estudos do tipo survey, segundo esse autor, a dependência entre observaçóes no nível micro acontece, muitas vezes, em virtude do uso freqüente de amostragens de grupos por áreas geográficas. Respondentes de uma mesma área geográfica tendem a ser mais similares do que respondentes de áreas geográficas diferentes. A homogeneidade entre as observaçóes conduz a estimativas erradas para os erros padrão. Esse tipo de erro é conhecido como o "efeito do delineamento" (Kish, 1965, 1987). Um procedimento de correção usualmente aplicado consiste em calcular os erros padrão por métodos de análise tradicionais, estimar a correlação intraclasse entre os respondentes dentro dos clusters, e, finalmente, empregar uma fórmula de correção para os erros padrão. Hox (2002) aponta que uma amostra de 200 alunos 
agrupados em 10 classes, com correlação intraclasse igual a 0,10, resulta, após a correção, em uma amostra de tamanho real igual a 69 alunos, ou seja, o tamanho factual da amostra diminuiu 66\%.

\section{ESTUDO NELS:88}

Em 1988, o governo norte-americano realizou a primeira etapa de uma extensa pesquisa longitudinal com estudantes da oitava série do primeiro ciclo educacional, intitulada National Education Longitudinal Study of 1988 (NELS:88). Amostras dos respondentes desta pesquisa foram submetidas a novos surveys em 1990, 1992, 1994 e 2000. No questionário inicial, os estudantes responderam sobre ampla gama de temas, incluindo experiências na escola, no trabalho e em casa; recursos e suporte educacional; o papel de seus pais e colegas em sua educação; características de sua vizinhança; anseios educacionais e profissionais; e outras percepçôes. Tópicos adicionais incluíam ainda autopercepção quanto ao tabagismo, uso de drogas e álcool e atividades extracurriculares (NCES, 1988).

Ita Kreft e Jan de Leeuw (1998), para ilustrar exemplos de seu livro, utilizaram parte dos dados do NELS:88, correspondentes a 21.580 estudantes de 1.003 escolas, tornando-os disponíveis em sua página na internet. Para este artigo, alguns dados foram recodificados em razão de sua origem categórica (como etnia) e outros em virtude do grande número de categorias presentes (como a relação alunos/professor). Também se utilizou uma codificação que originasse coeficientes positivos quando da geração dos modelos.

\section{OBJETIVO}

O presente artigo visa a ilustrar a elaboração de um modelo de regressão multinível, com base nos dados do NELS:88, tomando como variável dependente a nota obtida no teste de matemática.

\section{MÉTODO}

O modelo multinível da regressão para dois níveis é dado pela equação (1), conforme Hox (2002, p. 49):

$$
Y_{i j}=\gamma_{00}+\gamma_{p 0} X_{p i j}+\gamma_{0 q} Z_{q j}+\gamma_{p q} Z_{q j} X_{p i j}+u_{p j} X_{p i j}+u_{0 j}+e_{i j}
$$

O método utilizado para a elaboração do modelo, neste trabalho, é o mesmo apresentado por esse autor (p. 49-71). Tal método é composto de cinco passos, des- 
critos sumariamente a seguir. Para a elaboração dos modelos, usou-se a versão 2.0 do software MLwiN, e o método utilizado foi o IGLS - Iterative Generalised Least Squares (Rasbash et al., 2005).

\section{Passo 1}

Analisa-se um modelo sem nenhuma variável explicativa. Esse modelo, dito modelo somente de intercepto ou modelo vazio, é dado pela equação (2):

$$
Y_{i j}=\gamma_{00}+u_{0 j}+e_{i j}
$$

Na equação (2), $\gamma_{00}$ é o intercepto da regressão, e $u_{0 j}$ e $e_{i j}$ são os resíduos usuais nos níveis da escola (nível 2) e nível do aluno (nível 1), respectivamente. O modelo vazio é útil porque proporciona uma estimativa da correlaçáo intraclasse $\rho$ pela aplicação da equação (3):

$$
\rho=\frac{\sigma_{u 0}^{2}}{\left(\sigma_{u 0}^{2}+\sigma_{e}^{2}\right)}
$$

onde $\sigma_{u 0}^{2}$ é a variância dos resíduos $u_{0 j}$ do nível de escola, e $\sigma_{e}^{2}$ é a variância dos resíduos $e_{i j}$ do nível de aluno. O modelo vazio proporciona também uma medida de referência do deviance, o qual é uma medida do grau de desajuste do modelo e que pode ser usado para comparar modelos: quanto menor o deviance, maior o ajuste obtido.

\section{Passo 2}

Analisa-se um modelo com todas as variáveis explicativas fixas do nível mais baixo. Isso significa que os componentes de variância correspondentes aos coeficientes são fixados em zero. A decisão de inserir primeiramente as variáveis do nível mais baixo deve-se ao maior número de observaçóes disponíveis neste nível, gerando coeficientes mais acurados. Este modelo é descrito pela equação (4):

$$
Y_{i j}=\gamma_{00}+\gamma_{p 0} X_{p i j}+u_{0 j}+e_{i j}
$$

onde os $X_{p i j}$ são as $p$ variáveis explicativas do nível do aluno. Neste passo, estima-se a contribuição de cada variável explicativa deste nível.

\section{Passo 3}

Acrescentam-se as variáveis explicativas do nível da escola: 


$$
Y_{i j}=\gamma_{00}+\gamma_{p 0} X_{p i j}+\gamma_{0 q} Z_{q j}+u_{0 j}+e_{i j}
$$

onde os $Z_{q j}$ são as $q$ variáveis explicativas do nível da escola.

Os modelos dos passos 2 e 3 são chamados modelos de componentes de variância, por decomporem a variância do intercepto em componentes distintos de variância para cada nível hierárquico; nesses modelos, assume-se que o intercepto varia entre as escolas, mas os coeficientes de regressão são considerados fixos.

\section{Passo 4}

Avalia-se se algum dos coeficientes de regressão das variáveis explicativas do nível micro tem um componente significativo de variância (ou seja, diferente de zero) entre as escolas. Este modelo, chamado modelo de coeficientes randômicos, é dado pela equação (6):

$$
Y_{i j}=\gamma_{00}+\gamma_{p 0} X_{p i j}+\gamma_{0 q} Z_{q j}+u_{p j} X_{p i j}+u_{0 j}+e_{i j}
$$

onde os $u_{p j}$ são os resíduos do nível de escola dos coeficientes das variáveis explicativas $X_{p i j}$ do nível de aluno.

\section{Passo 5}

Adicionam-se as interações entre-níveis entre variáveis explicativas do nível da escola e aquelas variáveis explicativas do nível do aluno que tiveram variância significativa de coeficientes no passo 4. Isso conduz ao modelo completo formulado na equação (7):

$$
Y_{i j}=\gamma_{00}+\gamma_{p 0} X_{p i j}+\gamma_{0 q} Z_{q j}+\gamma_{p q} Z_{q j} X_{p i j}+u_{p j} X_{p i j}+u_{0 j}+e_{i j}
$$

Mais detalhes sobre os modelos de regressão multinível podem ser encontrados em Snijders e Bosker (1999), Hox (2002), Raudenbush e Bryk (2002), Rasbash et al. (2005), Tabachnick e Fidell (2007) e De Leeuw e Meijer (2008).

\section{RESULTADOS}

A construção do modelo vazio gerou os resultados exibidos na tabela 1. A correlaçáo intraclasse é dada por:

$$
\rho=\frac{\sigma_{u 0}^{2}}{\sigma_{u 0}^{2}+\sigma_{e}^{2}}=\frac{26,56}{(26,56+76,62)} \approx 0,26
$$


Tabela 1 - Modelo sem variáveis explicativas inseridas - modelo vazio (M1)

\begin{tabular}{l|ccc}
\hline \multicolumn{1}{c|}{ Variáveis explicativas } & \multicolumn{3}{c}{ Modelo 1 (M1) } \\
\hline Efeito fixo & Efeito & E.P. & Razão-t \\
\hline Intercepto & 50,80 & 0,18 & - \\
\hline
\end{tabular}

Efeito Randômico - nível $2-\sigma_{u 0}^{2}$

\begin{tabular}{|c|c|c|c|}
\hline Variância - intercepto & 26,56 & 1,36 & 19,53 \\
\hline \multicolumn{4}{|l|}{ Efeito Randômico - nível $1-\sigma_{e}^{2}$} \\
\hline Variância de $R_{i j}$ & 76,62 & 0,76 & 100,82 \\
\hline Correlação intraclasse & \multicolumn{3}{|c|}{0,26} \\
\hline Deviance M1 & \multicolumn{3}{|c|}{156.966} \\
\hline Número de parâmetros estimados no modelo & \multicolumn{3}{|c|}{3} \\
\hline
\end{tabular}

A tabela 1 indica um valor de 50,80 para o intercepto. Isso pode ser interpretado como a média geral dos alunos no teste de Matemática. A variância do intercepto mostra que esta média não é igual para todas as escolas. O teste de Wald revela que a variância do intercepto é significativamente diferente de zero, uma vez que a razão entre a variância e o erro-padrão (razão-t) é igual a 19,53. Este valor é consideravelmente superior ao valor crítico de 1,96 .

A variância $\mathrm{R}_{i j}$ (igual a 76,62) revela que dentro das escolas existe variabilidade entre os escores dos alunos no teste de Matemática. Uma vez mais, o teste de Wald mostra que esta variância é significativamente diferente de zero.

A correlação intraclasse de 0,26 significa que aproximadamente $26 \%$ da variância dos escores no teste de Matemática podem ser atribuídos ao nível da escola. $\mathrm{O}$ valor relativamente elevado da correlação intraclasse justifica a utilização de uma abordagem multinível de análise em vez de uma regressão tradicional.

O deviance, uma medida do desajuste do modelo em relação aos dados, não pode ser interpretrado isoladamente, mas sim comparado com os modelos posteriores. Espera-se que o ajuste melhore com a introdução de variáveis explicativas.

No modelo atual são estimados três parâmetros: o intercepto, a variância dos resíduos do nível da escola e a variância dos resíduos do nível do aluno. O número de parâmetros é utilizado para determinar o número de graus de liberdade para a comparação do ajuste do modelo.

Com a inserção das variáveis do nível do aluno, obtém-se o modelo $\mathrm{M} 2$, resumido na tabela 2. Foram inseridas as cinco variáveis seguintes: nível socioeconômico 
do aluno; etnia; horas gastas com o dever de casa, por semana; escolaridade dos pais e gênero. $\mathrm{O}$ modelo mostra que alunos de alto nível socioeconômico, de etnia asiática ou branca, do gênero masculino, com mais horas semanais dedicadas ao dever de casa, e pais com elevada escolaridade, apresentam melhor desempenho no teste de Matemática. Todos esses efeitos são significativos no nível de 5\%.

Observa-se sensível diminuição na variância do nível da escola, de 26,56 para 7,32. Isso se deve ao fato de que a proporção de cada uma das variáveis inseridas é desigualmente distribuída entre as escolas. Por exemplo, com relação ao gênero, existem escolas com proporçóes bastante variadas entre meninas e meninos.

De modo semelhante, também a variância do nível do aluno diminuiu no segundo modelo (de 76,62 para 66,07). Com a diminuiçáo da variância, tanto no nível da escola quanto no nível do aluno, a correlação intraclasse diminui de 0,26 para 0,10.

O deviance do modelo 2 é igual a 152.872, o que representa uma diminuição de 4.094 em relação ao modelo anterior. Foram estimados oito parâmetros no modelo 2, o que em comparação com os três parâmetros estimados no modelo 1 resulta em cinco graus de liberdade. O quociente dado pela diferença entre os deviances e os graus de liberdade é igual a 818,80 (teste qui-quadrado, representado pelo símbolo $\chi^{2}$ ). Este resultado significa que o modelo 2 se ajusta muito melhor aos dados do que o modelo 1 .

Com a introduçáo das variáveis do nível da escola, obteve-se o modelo M3, também resumido na tabela 2 .

Com a introdução das variáveis do nível socioeconômico agregado, etnia agregada, porcentagem de minorias raciais e região geográfica, a variância entre as escolas diminuiu de 7,32 para 4,89. Ao mesmo tempo, a variância entre os alunos apresentou pequena diminuiçáo de 66,07 para 65,92. Conseqüentemente, a correlaçáo intraclasse diminuiu de 0,10 para 0,07 .

O teste $\chi^{2}$, entre os modelos M2 e M3, resultou no valor de 77,00. Isso significa que o modelo M3 ajusta-se aos dados de modo significativamente melhor que o modelo M2.

A última informação da tabela 2 refere-se à variância explicada no nível do aluno e no nível da escola pelos modelos M2 e M3. Segundo Hox (2002, p. 64), a variância explicada no nível do aluno é dada pela equação:

$$
R_{1}^{2}=\left(\frac{\sigma_{e \mid b}^{2}-\sigma_{e \mid m}^{2}}{\sigma_{e \mid b}^{2}}\right)
$$


onde $\sigma_{e l b}^{2}$ é a variância residual do nível de aluno para o modelo de linha de base, que é o modelo vazio (M1), e $\sigma_{e \mid m}^{2}$ é a variância residual do nível de aluno no modelo de comparação (M2). Então:

$$
R_{1}^{2}=\left(\frac{76,62-66,07}{76,62}\right) \approx 0,138
$$

Tabela 2 - Modelos com as variáveis explicativas do nível 1 (M2) e do nível 2 (M3) inseridas

\begin{tabular}{|c|c|c|c|c|c|c|}
\hline \multirow{2}{*}{$\begin{array}{l}\text { Variáveis explicativas } \\
\text { Efeito fixo }\end{array}$} & \multicolumn{3}{|c|}{ Modelo 2 (M2) } & \multicolumn{3}{|c|}{ Modelo 3 (M3) } \\
\hline & Efeito & E.P. & Razão-t & Efeito & E.P. & Razão-t \\
\hline Intercepto & 43,71 & 0,33 & - & 41,29 & 0,77 & - \\
\hline $\begin{array}{l}\text { Nível socioeconômico do aluno } \\
\text { (NSE) }\end{array}$ & 3,16 & 0,15 & 21,07 & 2,69 & 0,15 & 17,93 \\
\hline Etnia (outras vs. asiático e branco) & 3,59 & 0,16 & 22,44 & 2,97 & 0,18 & 16,50 \\
\hline $\begin{array}{l}\text { Dever de casa (número de horas } \\
\text { semanais) }\end{array}$ & 1,23 & 0,04 & 30,75 & 1,20 & 0,04 & 30,00 \\
\hline Escolaridade dos pais & 0,70 & 0,09 & 7,78 & 0,67 & 0,09 & 7,44 \\
\hline Gênero & 0,52 & 0,11 & 4,73 & 0,51 & 0,11 & 4,64 \\
\hline Nível socioeconômico agregado & & & & 3,08 & 0,23 & 13,39 \\
\hline Etnia agregada & & & & 3,02 & 0,66 & 4,58 \\
\hline Porcentagem de minorias & & & & 0,23 & 0,08 & 2,88 \\
\hline Região (Sul e Oeste vs. demais) & & & & 0,54 & 0,19 & 2,84 \\
\hline \multicolumn{7}{|l|}{ Efeito Randômico - nível $2-\sigma_{u 0}^{2}$} \\
\hline Variância - intercepto & 7,32 & 0,47 & 15,57 & 4,89 & 0,36 & 13,58 \\
\hline \multicolumn{7}{|l|}{ Efeito Randômico - nível $1-\sigma_{e}^{2}$} \\
\hline Variância de $R_{i j}$ & 66,07 & 0,65 & 101,65 & 65,92 & 0,65 & 101,42 \\
\hline Correlação intraclasse condicional & & 0,10 & & & 0,07 & \\
\hline Deviance & & 152.872 & & & 152.564 & \\
\hline Número de parâmetros estimados & & 8 & & & 12 & \\
\hline Diferença Deviance M1 - Deviance M2 & & 4.094 & & & & \\
\hline Diferença de parâmetros M2 - M1 & & 5 & & & & \\
\hline Teste $\chi^{2}$ & & 818,80 & & & & \\
\hline Diferença Deviance M2 - Deviance M3 & & & & & 308 & \\
\hline Diferença de parâmetros M3 - M2 & & & & & 4 & \\
\hline Teste $\chi^{2}$ & & & & & 77,00 & \\
\hline Variância do nível 1 explicada & & $13,8 \%$ & & & $14,0 \%$ & \\
\hline Variância do nível 2 explicada & & $72,4 \%$ & & & $81,6 \%$ & \\
\hline
\end{tabular}


De acordo com Hox (2002, p. 64), para a proporção da variância explicada no nível da escola, usa-se:

$$
R_{2}^{2}=\left(\frac{\sigma_{u 0 \mid b}^{2}-\sigma_{u 0 \mid m}^{2}}{\sigma_{u 0 \mid b}^{2}}\right)
$$

onde $\sigma_{u 0 \mid b}^{2}$ é a variância residual do nível de escola para o modelo de linha de base (M1), e $\sigma_{u 0 \mid m}^{2}$ é a variância residual do nível de escola para o modelo de comparação (M2). Então:

$$
R_{2}^{2}=\left(\frac{26,56-7,32}{26,56}\right) \approx 0,724
$$

Para o modelo com as variáveis explicativas do nível de escola (M3), tem-se, para o nível do aluno:

$$
R_{1}^{2}=\left(\frac{76,62-65,92}{76,62}\right) \approx 0,139
$$

Ainda no modelo M3, para o nível da escola tem-se:

$$
R_{2}^{2}=\left(\frac{26,56-4,89}{26,56}\right) \approx 0,816
$$

Os modelos de coeficientes randômicos (M4) e de interações entre variáveis dos níveis 1 e 2 (M5) são mostrados na tabela 3.

Quatro variáveis do nível do aluno apresentaram um coeficiente randômico significativo, ou seja, o efeito dos coeficientes de NSE, etnia, dever de casa e gênero náo é igual entre todas as escolas. Por exemplo, a influência do nível socioeconômico do aluno sobre o seu desempenho no teste de matemática é menor em algumas escolas do que em outras.

O teste $\chi^{2}$, entre os modelos M3 e M4, resultou no valor de 7,21. Isto indica que o modelo, depois da inclusáo dos efeitos randômicos, se ajusta melhor aos dados, em comparaçáo com o modelo anterior.

A única interação entre variáveis dos níveis micro e macro a apresentar um valor significativamente diferente de zero foi a interação entre NSE e etnia agregada. A inclusão desta interação no modelo, resultando no modelo final (M5), melhorou o ajuste, conforme indica o valor do teste $\chi^{2}$ com valor igual a 48,00. 
Tabela 3 - Modelos de coeficientes randômicos (M4) e com interações entre os níveis 1 e 2 (M5)

\begin{tabular}{|c|c|c|c|c|c|c|}
\hline \multirow{2}{*}{$\begin{array}{l}\text { Variáveis explicativas } \\
\text { Efeito fixo }\end{array}$} & \multicolumn{3}{|c|}{ Modelo 4 (M4) } & \multicolumn{3}{|c|}{ Modelo 5 (M5) } \\
\hline & Efeito & E.P. & Razão-t & Efeito & E.P. & Razão-t \\
\hline Intercepto & 41,32 & 0,77 & - & 40,49 & 0,78 & - \\
\hline Nível socioeconômico do aluno (NSE) & 2,65 & 0,15 & 17,67 & 1,16 & 0,26 & 4,46 \\
\hline Etnia (outras vs. asiático e branco) & 2,93 & 0,20 & 14,65 & 3,02 & 0,20 & 15,10 \\
\hline Dever de casa (n. de horas semanais) & 1,20 & 0,04 & 30,00 & 1,19 & 0,04 & 29,75 \\
\hline Escolaridade dos pais & 0,67 & 0,09 & 7,44 & 0,63 & 0,09 & 7,00 \\
\hline Gênero & 0,52 & 0,13 & 4,00 & 0,53 & 0,13 & 4,08 \\
\hline Nível socioeconômico agregado & 3,15 & 0,23 & 13,70 & 2,96 & 0,23 & 12,87 \\
\hline Etnia agregada & 2,99 & 0,67 & 4,46 & 3,97 & 0,68 & 5,84 \\
\hline Porcentagem de minorias & 0,23 & 0,08 & 2,88 & 0,24 & 0,08 & 3,00 \\
\hline Região (Sul e Oeste vs. demais) & 0,58 & 0,19 & 3,05 & 0,52 & 0,19 & 2,74 \\
\hline NSE $x$ etnia agregada & & & & 2,10 & 0,30 & 7,00 \\
\hline \multicolumn{7}{|l|}{ Efeito Randômico - nível $2-\sigma_{u 0}^{2}$} \\
\hline Variância - intercepto & 7,77 & 1,27 & 6,12 & 7,25 & 1,23 & 5,89 \\
\hline Variância - inclinação NSE & 0,73 & 0,34 & 2,15 & 0,48 & 0,33 & 1,44 \\
\hline Variância - inclinação etnia & 5,40 & 1,25 & 4,32 & 5,23 & 1,23 & 4,25 \\
\hline Variância - inclinação dever de casa & 0,33 & 0,08 & 4,13 & 0,33 & 0,08 & 4,13 \\
\hline Variância - inclinação gênero & 3,73 & 0,73 & 5,11 & 3,71 & 0,73 & 5,11 \\
\hline \multicolumn{7}{|l|}{ Efeito Randômico - nível $1-\sigma_{e}^{2}$} \\
\hline Variância de $R_{i j}$ & 63,54 & 0,67 & 94,84 & 63,77 & 0,66 & 96,62 \\
\hline Deviance & & 152.463 & & & 152.415 & \\
\hline Número de parâmetros estimados & & 26 & & & 27 & \\
\hline Diferença Deviance M3 - Deviance M4 & & 101 & & & & \\
\hline Diferença de parâmetros M4 - M3 & & 14 & & & & \\
\hline Teste $\chi^{2}$ & & 7,21 & & & & \\
\hline Diferença Deviance M4 - Deviance M5 & & & & & 48 & \\
\hline Diferença de parâmetros M5 - M4 & & & & & 1 & \\
\hline Teste $\chi^{2}$ & & & & & 48,00 & \\
\hline
\end{tabular}

$\mathrm{Na}$ tabela 3 não constam os valores das variâncias explicadas dos níveis micro e macro. Isso se deve ao fato de que a decomposiçáo da variância com a presença de coeficientes randômicos e de interaçôes entre os níveis é extremamente complexa (Snijders, Bosker, 1999, p. 108).

A diferença entre os modelos M4 e M5 é a presença do efeito de interação entre as variáveis NSE do aluno e etnia agregada. O efeito de interação pode ser resumido 
pela afirmação efeitos diferentes para grupos diferentes (Miles, Shevlin, 2001, p. 165). Neste caso, a influência do nível socioeconômico do aluno é diferente entre escolas de etnia predominantemente branca e asiática versus as demais etnias.

Observe-se ainda o efeito da recodificação empregada, a qual produziu coeficientes de regressão positivos. Especificamente quanto ao gênero (codificado na forma 0 = meninas e 1 = meninos), depreende-se que os meninos têm desempenho médio geral, no teste de matemática, superior ao desempenho médio geral das meninas. Raciocínio análogo pode ser empregado no tocante à variável região, em que a recodificação permite avaliar que o desempenho médio geral das regióes Sul e Oeste (codificadas com o valor 0) é inferior ao desempenho médio geral das regióes Nordeste e Centro-norte (codificadas com o valor 1), no teste de matemática.

O teste $\chi^{2}$, entre os modelos M1 e M5, mostra o seguinte resultado:

$$
\frac{\text { Deviance } M 1-\text { Deviance } M 5}{\text { Parâmetros } M 5-\text { Parâmetros } M 1}=\frac{156.966-152.415}{27-3} \approx 189,62
$$

Uma vez que o teste $\chi^{2}$ resultou em um valor muito superior ao valor crítico de 1,96, fica claramente demonstrado que o modelo final se ajusta muito melhor aos dados do que o modelo inicial.

Também merece comentário a relação entre o valor e o erro-padrão dos coeficientes randômicos da variável nível socioeconômico do aluno (NSE), no modelo M5. Embora essa relaçáo seja igual a 1,44 (tabela 3), portanto não significativa para um intervalo de confiança de $95 \%$, considerou-se que o deviance do modelo como um todo foi melhorado, decidindo-se desse modo pela permanência desses coeficientes no modelo. Essa decisão é baseada na recomendação de Kreft e Leeuw (1998) de que nas ciências sociais, com alto grau de inter-relação de variáveis, é mais adequado avaliar o ajuste do modelo como um todo do que depender da razáo-t de variáveis individuais.

\section{VERIFICAÇÃO DE PRESSUPOSTOS}

Um dos pressupostos principais da análise multinível é a distribuição normal dos resíduos de cada nível. A normalidade pode ser verificada por meio de um gráfico mostrando os resíduos ordenados versus escores gerados aleatoriamente em uma distribuição normal. Se o pressuposto da normalidade é válido, os pontos no gráfico estaráo dispostos em uma linha reta. A figura 1 ilustra o gráfico dos resíduos do nível 1 para o modelo final (M5). Uma vez que nesta análise multinível existem resíduos em dois níveis, a verificaçáo também deve ser repetida para o segundo nível. 
O gráfico mostra que os resíduos se distribuem linearmente ao longo dos valores, sem a presença de outliers extremos, ou seja, o modelo de regressão linear é adequado, com os resíduos de nível 1 seguindo uma distribuição bastante aproximada da normal.

Figura 1 - Resíduos padronizados X escores normais para o modelo M5

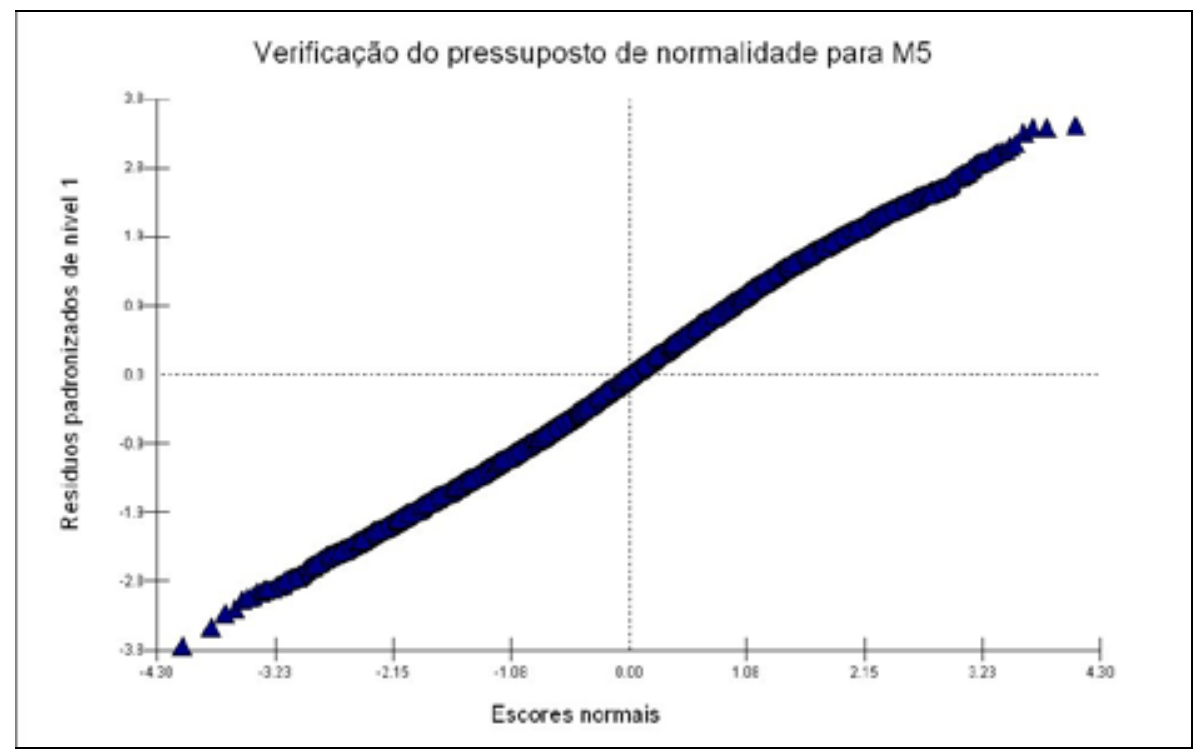

A figura 2 ilustra o gráfico dos resíduos padronizados $\mathrm{X}$ valores preditos para o modelo final. Como os resíduos são distribuídos de modo uniforme em torno da média (zero), o gráfico mostra que os pressupostos de normalidade, linearidade e homocedasticidade não são violados pelo modelo gerado (Raudenbush; Bryk, 2002, p. 131).

\section{CONCLUSÕES}

O banco de dados do NELS:88 mostrou-se adequado para ilustrar a utilização de uma análise multinível. Primeiramente, os dados não violam os pressupostos da análise multinível. Além disso, o banco possui um número elevado de unidades de ambos os níveis. Muitas vezes, em situaçóes práticas, o nível macro náo possui um número mínimo necessário de unidades, o qual, segundo Kreft (1996), seria de 30 a 50. Também existem variáveis em número suficiente, com efeitos 
significativos em ambos os níveis. Por fim, o banco de dados permite identificar um efeito de interação.

Figura 2 - Resíduos padronizados X escores preditos para o modelo M5

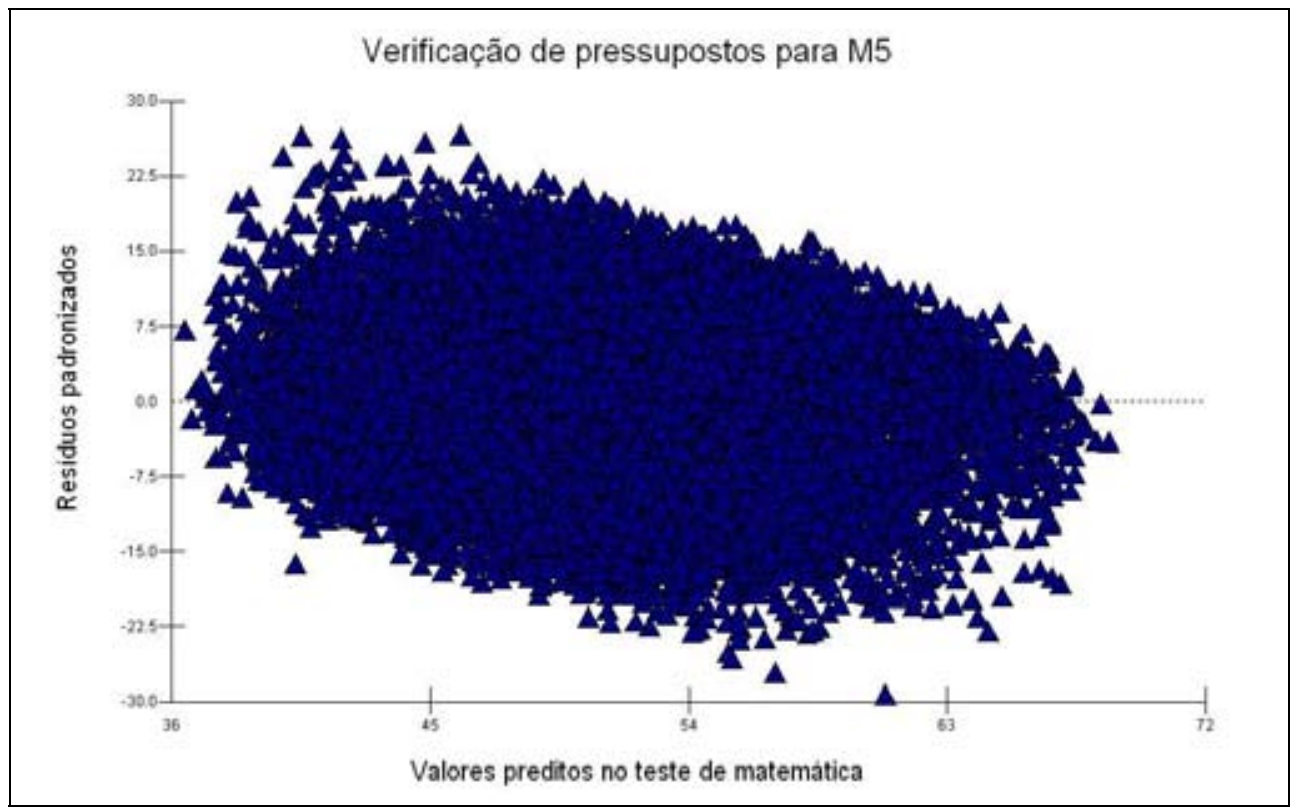

\section{REFERÊNCIAS BIBLIOGRÁFICAS}

DE LEEUW, J.; MEIJER, E. Handbook of multilevel analysis. New York, NY: Springer Science + Business Media, 2008.

HOX, J. Multilevel analysis: techniques and applications. Mahwah, NJ: Lawrence Erlbaum Associates, 2002.

$\mathrm{KISH}$, L. Statistical design for research. New York: Wiley, 1987.

1965.

. Survey sampling. New York: Wiley,

KREFT, I. G. G. Are multilevel techniques necessary? An overview, including simulation studies. 1996. Disponível em: <http://ioe.ac.uk/ multilevel> Acesso em: 23 out. 2005.
KREFT,I.G.G.;DELEEUW, J.Introducingmultilevel modeling. 1998. Disponível em: < http://www. stat.ucla.edu/ deleeuw/sagebook $>$ Acesso em: 23 out. 2005.

MILES, J.; SHEVLIN, M. Applying regression and correlation: a guide for students and researchers. London: Sage Publications, 2001. NCES. National Education Longitudinal Study of 1988 (Rel. Tec.). Washington: National Center for Education Statistics, 1988. Disponível em: $<$ http://nces.ed.gov/surveys/nels88>. Acesso em: 14 nov. 2005.

RASBASH, J. et al. A User's guide to MLwiN $v$. 2.0. London. Centre for Multilevel Modelling. 
University of Bristol, UK, 2005. RAUDENBUSH, S. W.; BRYK, A. S. Hierarchical Linear Models: applications and data analysis methods. Thousand Oaks: Sage Publications, 2002.

SNIJDERS, T. A. B.; BOSKER, R. J. Multilevel analysis: an introduction to basic and advanced multilevel modeling. London: Sage Publications, 1999.

TABACHNICK, B. G.; FIDELL, L. S. Multilevel Linear Modeling. In: (orgs.) Using Multivariate Statistics. Boston: Pearson Education, 2007. p.781-857.

Recebido em: setembro 2007

Aprovado para publicação em: março 2008

Jacob Arie Laros é Professor da Pós-graduação em Psicologia Social do Trabalho e das Organizaçôes (PSTD) da Universidade de Brasília (jalaros@unb.br); João Luiz Pereira Marciano é doutor em Ciência da Informação na Universidade de Brasília (joao.marciano@camara.gov.br). 